Article

\title{
Prospective Use of High-Refractive Index Materials for Single Molecule Detection in Flow Cytometry
}

\author{
Joshua A. Welsh ${ }^{1}{ }^{(1)}$, Julia Kepley ${ }^{1}$, Ariel Rosner ${ }^{1}$, Peter Horak ${ }^{2}{ }^{(D)}$, Jay A. Berzofsky ${ }^{1}$ (D) and \\ Jennifer C. Jones ${ }^{1, *(1)}$ \\ 1 Vaccine Branch, Center for Cancer Research, National Institutes of Health, Bethesda, MD 20892, USA; \\ joshua.welsh@nih.gov (J.A.W.); julia.kepley@nih.gov (J.K.); arijrosner@gmail.com (A.R.); \\ berzofsj@mail.nih.gov (J.A.B.) \\ 2 Optoelectronics Research Centre, University of Southampton, Southampton SO17 1BJ, UK; \\ peh@orc.soton.ac.uk \\ * Correspondence: jennifer.jones2@nih.gov; Tel.: +1-301-496-5457
}

Received: 23 April 2018; Accepted: 18 July 2018; Published: 1 August 2018

\begin{abstract}
Phenotyping extracellular vesicles (EVs), where surface receptor expression is often as low as one molecule per $\mathrm{EV}$, remains problematic due to the inability of commercial flow cytometers to provide single-fluorescent molecule sensitivity. While EVs are widely considered to be of great potential as diagnostic, prognostic and theranostic biomarkers, their use is currently hindered by the lack of tools available to accurately and reproducibly enumerate and phenotype them. Herein, we propose a new class of labels that leverage the biophysical properties of materials with unique complex refractive indices and demonstrate that this class of labels has the possibility of allowing single-epitope detection using conventional flow cytometry.
\end{abstract}

Keywords: extracellular vesicles; flow cytometry; light scatter; scatter modelling; complex refractive index; scatter labels; NanoTags; single-epitope detection

\section{Introduction}

Extracellular vesicles (EVs) are small (30-1000 nm) membranous vesicles with the majority being $<150 \mathrm{~nm}$ in diameter [1]. Due to their small surface area, when compared to cells, the epitope expression of the majority of EV surface markers is below the detectable range of conventional high throughput, multi-parameter detection techniques, such as flow cytometry [2].

Conventional flow cytometry ( $\mathrm{cFC}$ ) is capable of distinguishing thousands, to a few hundred, fluorescent molecules. The surface expression of highly abundant lymphocyte surface marker, such as CD14, is in the region of 100,000 copies per lymphocyte. If this epitope density were scaled to a $100 \mathrm{~nm}$ EV, it would equate to <30 CD14 copies per vesicle [3]. Therefore, an unmet need in the EV field is the development of labels that enable single-epitope detection on single EVs, ideally utilizing conventional detection equipment.

cFC quantifies the expression of proteins using fluorescent probes, which tend to be in the form of fluorescently conjugated antibodies. While these have proven to be a powerful tool for cellular analysis in the immunology field, in their current form they are insufficient to quantify EV surface protein expression on the majority of available flow cytometers. While newer generations of fluorescent labels have emerged, such as QDots, they remain generally inadequate for low surface epitope quantification. The use of QDots as labels for time-limited detection of individual targets is also complicated by stochastic optical fluctuation, commonly referred to as "blinking" [4].

When the objective of an analysis is to enumerate the number of EVs which are positive for a specific marker in a sample, the method of enumeration must be able to detect each EV with one 
or more specific surface marker. In this case, it is essential to use labels and instruments capable of detecting single labels, with single label sensitivity. On the other hand, when the objective is to quantify or compare how many receptors are present on individual EVs, there must be a constant label-to-target ratio and labels with monovalent target binding-sites are needed for single molecule detection and surface receptor quantification on EVs. Other standardization steps should also be considered when enumerating EVs, such as dilution controls, MESF beads, and/or scatter modelling [5]. Most commercially available techniques utilizing QDots use these labels in polyvalent forms but new avenues have emerged that have attempted to create monovalent QDots which may be directly transferrable to the conjugation of other nanoparticles [6-8].

Here, we propose the development of a novel class of nanoscale molecular tags (NanoTags) that could enable single label and therefore single molecule, detection using conventional flow cytometers. These NanoTags would be composed of materials with high refractive indices and/or high optical absorption and unique spectral scattering properties that would enable both low epitope number enumeration and spectral phenotyping of small particles, such as extracellular vesicles.

In this paper, we shall demonstrate how NanoTag compositions may be selected for unambiguous optical detection and how multiple NanoTag compositions could be used simultaneously to allow particle phenotyping and still be distinguished from one another. Our analysis is based on numerical modelling of wavelength dependent light scattering by nanoparticles and comparison with flow cytometry data.

\section{Materials and Methods}

\subsection{Particle Refractive Index}

To first identify particles with useful optical properties that would aid increased light scattering, the refractive index and extinction coefficients were collated, Supplementary Figure S1. Particle refractive indices and extinction coefficients across wavelengths $300-800 \mathrm{~nm}$ were compiled from the literature for the following compositions: gold, silver, iron oxide, titanium dioxide, copper, platinum, lead and zirconium [9-13]. The refractive indices of polystyrene and water were calculated at each wavelength using the corresponding Sellmeier equations [14,15].

The compositions selected were narrowed down by with the inclusion criteria of being currently sold in a nanoparticle format and having a high refractive index (e.g., Titanium Dioxide) or extinction efficient (e.g., Lead) or having a medium refractive index and extinction coefficient (e.g., Gold, Silver, Copper).

\subsection{Particle Analysis Using Flow Cytometry}

Flow cytometry measurements were performed on a MoFlo Astrios EQ (Beckman Coulter Life Sciences, Indianapolis, IN, USA) and FACS Symphony (BD Life Sciences, Franklin Lakes, NJ, USA) flow cytometers. The Astrios EQ is a jet-in-air system with 5 lasers $(355,405,488,561$ and $640 \mathrm{~nm}$ wavelength), with side scatter collection (SSC) detection possible at 405, 488, 561 and $640 \mathrm{~nm}$. Detailed description of Astrios EQ setup and detection thresholds can be found in the literature [16]. The FACS Symphony is a cell-analyzer with 5 lasers $(355,405,488,532$ and $640 \mathrm{~nm})$ with SSC detection only possible at $488 \mathrm{~nm}$ with the default device configuration. The equipment parameters for particle enumeration via SSC were set to a triggering threshold of 200, a voltage of 350 and a low fluidics rate.

For measurements of scattered power versus diameter, NIST-traceable polystyrene beads (100, 125, 147, 203, 296, 400, 600, 799, 994 nm diameter) (Thermo Fisher Scientific, gift from E. van der Pol, Waltham, MA, USA) and silica beads $(182,315,359,405,548,800,1000 \mathrm{~nm})$ (Kisker Biotech, gift from E. van der Pol, Amsterdam, The Netherlands) were analyzed at a concentration of $0.1-1 \times 10^{8}$ particles per $\mathrm{mL}$ and confirmed the fit of predicted scatter versus acquired scatter using previously published flow cytometer methodology [17]. 
For high-refractive index particle acquisition modelling comparisons, Ag particles, 20, 40, 60, 80 and $100 \mathrm{~nm}$ in diameter (Cytodiagnostics, Burlington, ON, Canada), Au particles, 20, 40, 60 and $80 \mathrm{~nm}$ in diameter (Cytodiagnostics) and $100 \mathrm{~nm}$ polystyrene NIST-traceable beads (Thermo Fisher Scientific) and $200 \mathrm{~nm}$ fluorescent polystyrene beads (Thermo Fisher Scientific) were diluted to a concentration between $0.1-1 \times 10^{7}$ particles per $\mathrm{mL}$ before acquisition on each instrument. Linear dilutions of 40, 60, $80 \mathrm{~nm}$ Au particles were performed to confirm working cytometer acquisition concentration that maintained single-particle detection (Supplementary Figure S2). Particle concentrations were determined using manufacturer percentage solids. Spike-in particle concentrations were determined using nanoparticle tracking analysis.

Data was acquired using the acquisition software Summit v6 on the Astrios EQ and Diva v8 for the FACS Symphony. Upon completion of data acquisition, files were exported from the acquisition software and imported into FlowJo v10 (TreeStar) for post-acquisition analysis. Flow cytometry files can be found at: https:/ / flowrepository.org/id/FR-FCM-ZYL7 and https:/ / flowrepository.org/id/ FR-FCM-ZYL6.

\subsection{Flow Cytometer and Particle Spectral Scatter Modelling}

All numerical modelling was performed with MATLAB v9.3.0 (The MathWorks Inc., Natick, MA, USA). The cumulative power of light scattered by a spherical particle of fixed diameter $(20,40,60$ $80,100 \mathrm{~nm}$ ), reaching a detector was calculated using Mie theory and implemented numerically with scripts that built upon those from Matzler [18]. The calculations used resemble those of van der Pol et al. [14] and Fattaccioli et al. [15]. This software is available at: http:/ / www.joshuawelsh. co.uk/scatter-diameter-software/. Particle light scatter modelling focused on the side scatter, that is, on light collected perpendicular to the direction of illumination. The side scatter collection optics of the Astrios EQ flow cytometer have a circular collection aperture with a limiting half-angle approximated to be $29^{\circ}[17,19]$. The particle suspension medium was modelled using the optical properties of water. Fitting of the modelled data to the experimental data, in order to produce curves of scattered power (or equivalently, scattering cross section) versus particle diameter, was carried out using the method of van der Pol et al. [14] using a single scale factor to convert between simulated scattering cross sections and experimentally measured power levels. Flow cytometry data was converted from arbitrary units to scattering cross-section in $\mathrm{nm}^{2}$ using linear regression of predicted versus acquired values from modelling (Supplementary Figure S3).

\subsection{Particle Analysis Using Nanoparticle Tracking Analysis (NTA)}

Gold and silver particle diameter distributions and concentrations were measured using NTA with a NanoSight LM10 instrument (Malvern, UK), equipped with a $405 \mathrm{~nm}$ LM12 module and EMCCD camera (DL-658-OEM-630, Andor, Belfast, UK). Video acquisition was performed with NTA software v3.2, using a camera level of 14 . Three $30 \mathrm{~s}$ videos were captured per sample. Post-acquisition video analysis used the following settings: minimum track length $=5$, detection threshold $=4$, automatic blur size $=2$-pass, maximum jump size $=12.0$.

\section{Results}

\subsection{Spectral Scatter Modelling of Different Particle Compositions}

Using previously developed methods of flow cytometer particle scatter modelling, Figure 1 combined with reference refractive index and extinction coefficients from the literature, it is possible to use these results, so as to predict which particle diameters and compositions may be detectable. Here, the side scatter collection optics of the Astrios EQ cytometer have been modelled to determine the relative detected scattering power of $\mathrm{Au}$ and Ag nanoparticles across the UV-visible spectrum, Figure 2. Other compositions (platinum, titanium dioxide, iron oxide, copper, lead, zirconium) were also modeled, Supplementary Figure S4. 
The green lines represent the range of EV refractive indices (inner refractive index $n=1.38 \pm 0.02$, membrane refractive index $n=1.48$ and a membrane diameter of $10 \mathrm{~nm}$ ). The scattering power of $100 \mathrm{~nm}$ and $1000 \mathrm{~nm}$ EVs, assuming an average refractive index, are also shown (black lines). Instrument noise floor statistics are also displayed (blue lines).

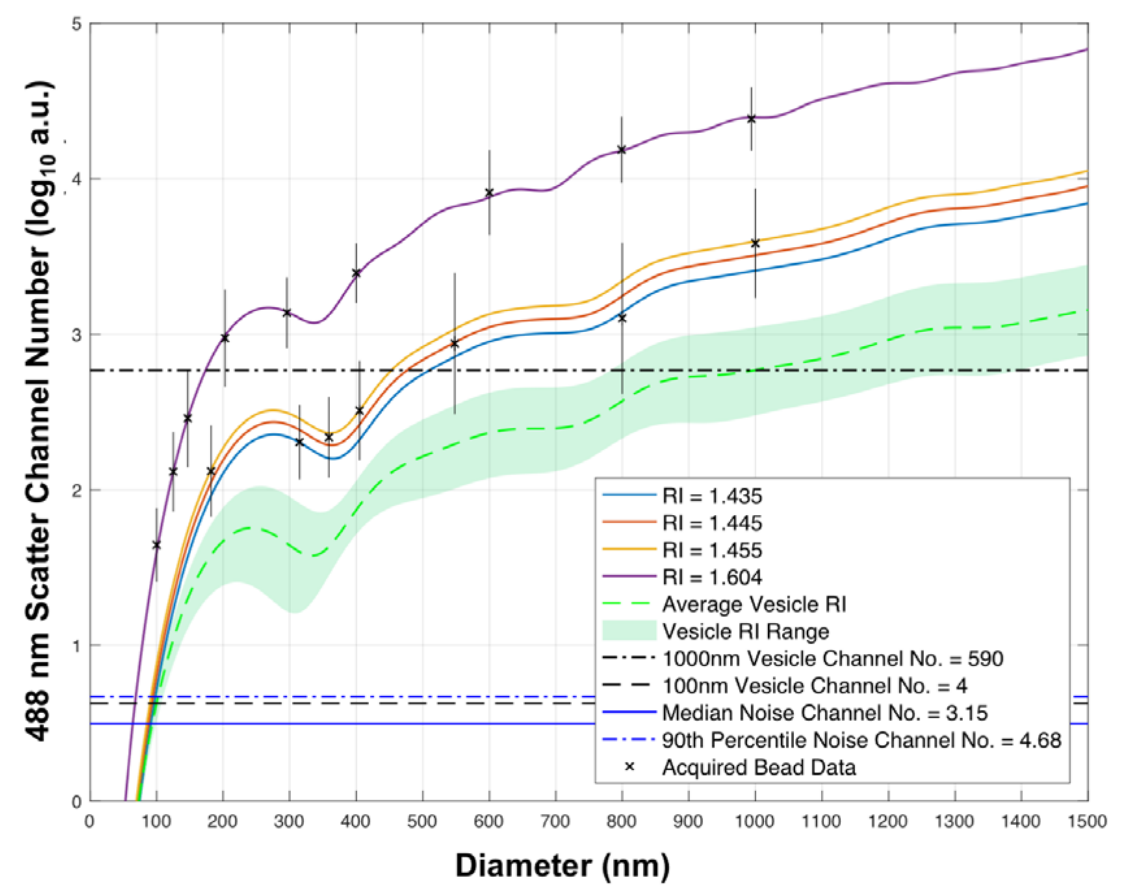

Figure 1. Scatter-diameter relationship of acquired vs. predicted polystyrene and silica particles on Astrios EQ. Predicted scattering cross-sectional units were normalized to flow cytometer arbitrary unit data using a single normalization factor. Error bars show the range of the acquired bead scattering in arbitrary units, with crosses indicating median acquired scattering of each bead.

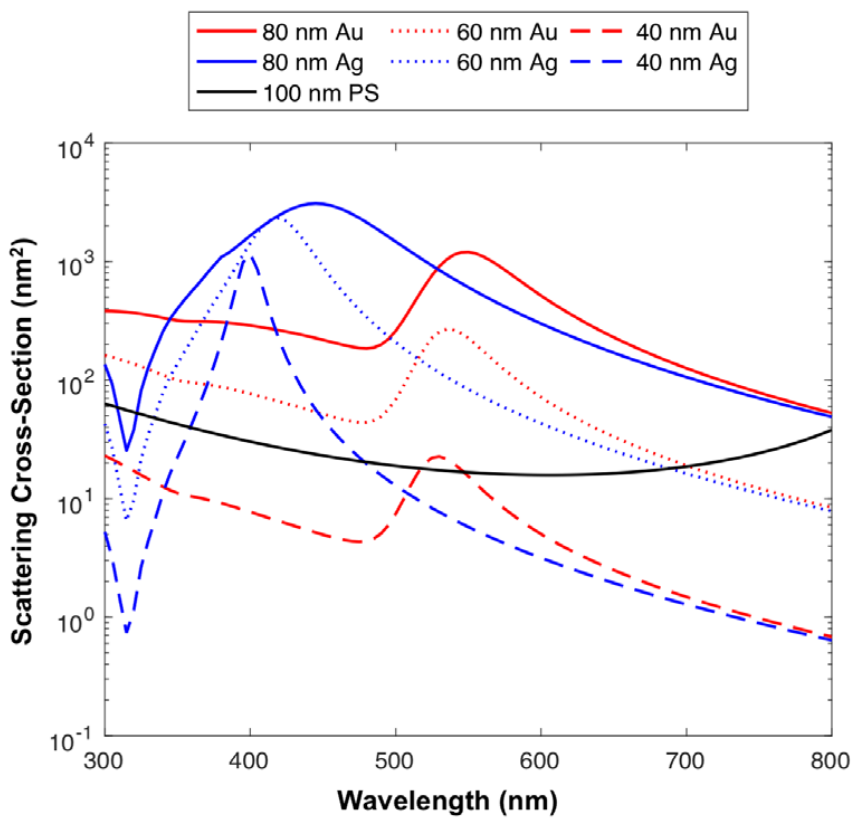

Figure 2. Modelled scattering cross-sections assuming Astrios EQ collection optics. Displayed are the scattering cross-sections of $40 \mathrm{~nm}, 60 \mathrm{~nm}, 80 \mathrm{~nm}$ Au (red) and Ag (blue) spheres and $100 \mathrm{~nm}$ polystyrene spheres (black). 
Using $100 \mathrm{~nm}$ polystyrene (PS) beads as a cross-sectional scattering reference, due to them being detectable on a number of cFC platforms it can be seen that $20 \mathrm{~nm}$ silver nanoparticles illuminated at $\sim 400 \mathrm{~nm}$ have a higher scattering cross-section than $100 \mathrm{~nm}$ PS particles, Figure 2. $40 \mathrm{~nm} \mathrm{Au}$ particles surpass the scattering cross-section of $100 \mathrm{~nm}$ PS sphere at an illumination wavelength of $\sim 532 \mathrm{~nm}$, with $40 \mathrm{~nm}$ Ag particles having a high scattering cross-section with illumination wavelengths ranging from $\sim 350-450 \mathrm{~nm}$, Figure $2.60 \mathrm{~nm}$ spheres of all compositions with the exception of cadmium selenide, iron oxide and titanium dioxide, have a higher scattering cross-section than $100 \mathrm{~nm}$ PS spheres across the majority of the UV-visible spectrum, before dropping in the red area $(>700 \mathrm{~nm})$ of the spectrum, Figure 2.

\subsection{Modelled Particle Spectral Scattering versus Acquired Particle Spectral Scattering}

PS, Au and Ag nanoparticles were analyzed on the Astrios EQ (Figure 3) and FACS Symphony (Supplementary Figure S5) instrument to determine whether particles were detectable using the conventional scatter collection wavelength of $488 \mathrm{~nm}$, Figure 3. Unstained $100 \mathrm{~nm}$ PS beads were resolvable on both instruments. 100, 80, 60 and $40 \mathrm{~nm} \mathrm{Ag} \mathrm{particles} \mathrm{were} \mathrm{distinguishable} \mathrm{from}$ background on both instruments, with the $40 \mathrm{~nm}$ population being only partially resolved on the Astrios EQ and FACS Symphony from background noise. $20 \mathrm{~nm} \mathrm{Ag}$ or Au particles were not resolved on either instrument. 80, 60 and $40 \mathrm{~nm}$ Au particles were resolved on both instruments, again with $40 \mathrm{~nm}$ Au being only partially resolved on each instrument. We note that these results are consistent with the simulations of Figure 2; at $488 \mathrm{~nm}$ wavelength for all sizes $\mathrm{Au}$ and Ag particles have similar scattering cross sections, with Ag having the slightly larger cross section as is also evident in the measured data.

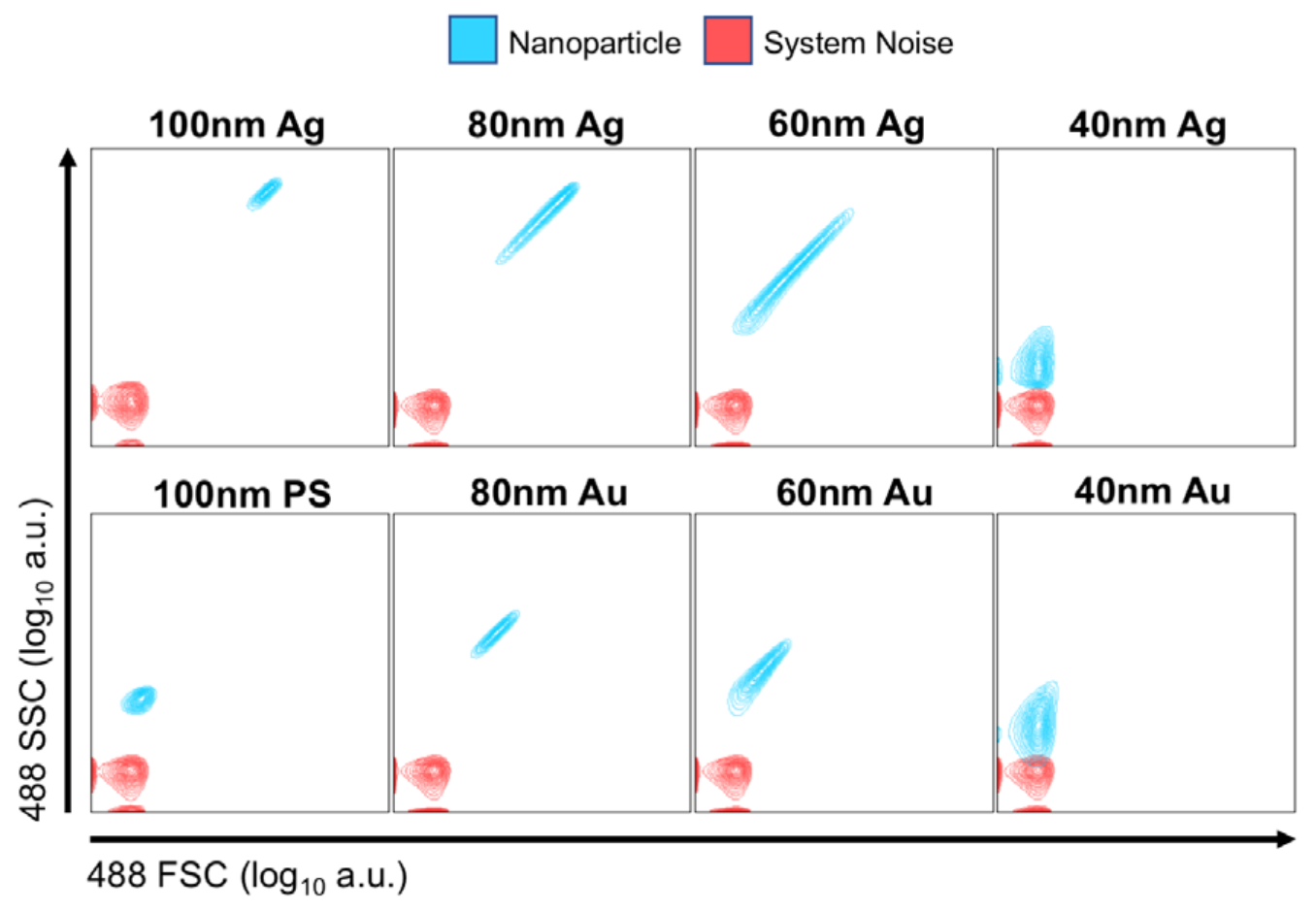

Figure 3. Comparison of the scattered optical power at $488 \mathrm{~nm}$ wavelength for gold ( $\mathrm{Au}$ ), silver (Ag) and polystyrene (PS) nanoparticles measured on Astrios EQ in the forward (FSC) and sideward (SSC) direction. Power levels are plotted on a logarithmic scale. The Astrios EQ instrument collects particle scattering at multiple wavelengths allowing quantification of system noise which is color coded. This is not easily done using the FACS Symphony and hence it is not color coded. 
The PS, Au and Ag particles were then investigated using multi-wavelength modelling of the Astrios EQ, Figure 4A and comparing them to the acquired data from 488 and $561 \mathrm{~nm}$ SSC channels, Figure 4B. A comparison between modelled and acquired data at 405, 488, 561, $640 \mathrm{~nm}$ can be found in Supplementary Figure S6. Using the instrument background noise as a reference, $40 \mathrm{~nm}$ Ag particles were partially resolved on $561 \mathrm{~nm}, 488 \mathrm{~nm}$ and $405 \mathrm{~nm}$ SSC channels and unresolved on the $640 \mathrm{~nm}$ SSC channel. $40 \mathrm{~nm}$ Au particles were fully resolved on the $561 \mathrm{~nm}$ and $488 \mathrm{~nm}$ SSC channels, partially resolved on $640 \mathrm{~nm}$ SSC channel and unresolved on $405 \mathrm{~nm}$ SSC channel. $100 \mathrm{~nm}$ PS particles were fully resolved on $488 \mathrm{~nm}$ and $561 \mathrm{~nm}$ SSC channels, partially resolved on the $405 \mathrm{~nm}$ SSC channel and unresolved on the $640 \mathrm{~nm}$ SSC channel. All other particles were resolvable from instrument background noise on all SSC channels.

The relationship between $\mathrm{Au}, \mathrm{Ag}$ and PS particle scattering on all channels was well maintained between models and acquired data, Figure 4A,B, Au particles show a linear increase in scattering between $488 \mathrm{~nm}$ and $561 \mathrm{~nm}$ SSC channels, with the PS particles appearing to also increase linearly but with increased $488 \mathrm{~nm}$ scattering than Au and finally Ag appearing to scatter linearly between $488 \mathrm{~nm}$ and $561 \mathrm{~nm}$ scattering channels before beginning to tail off in scattering on the $488 \mathrm{~nm}$ scattering channel between $60-80 \mathrm{~nm}$ but continue to increase in $561 \mathrm{~nm}$ scattering.
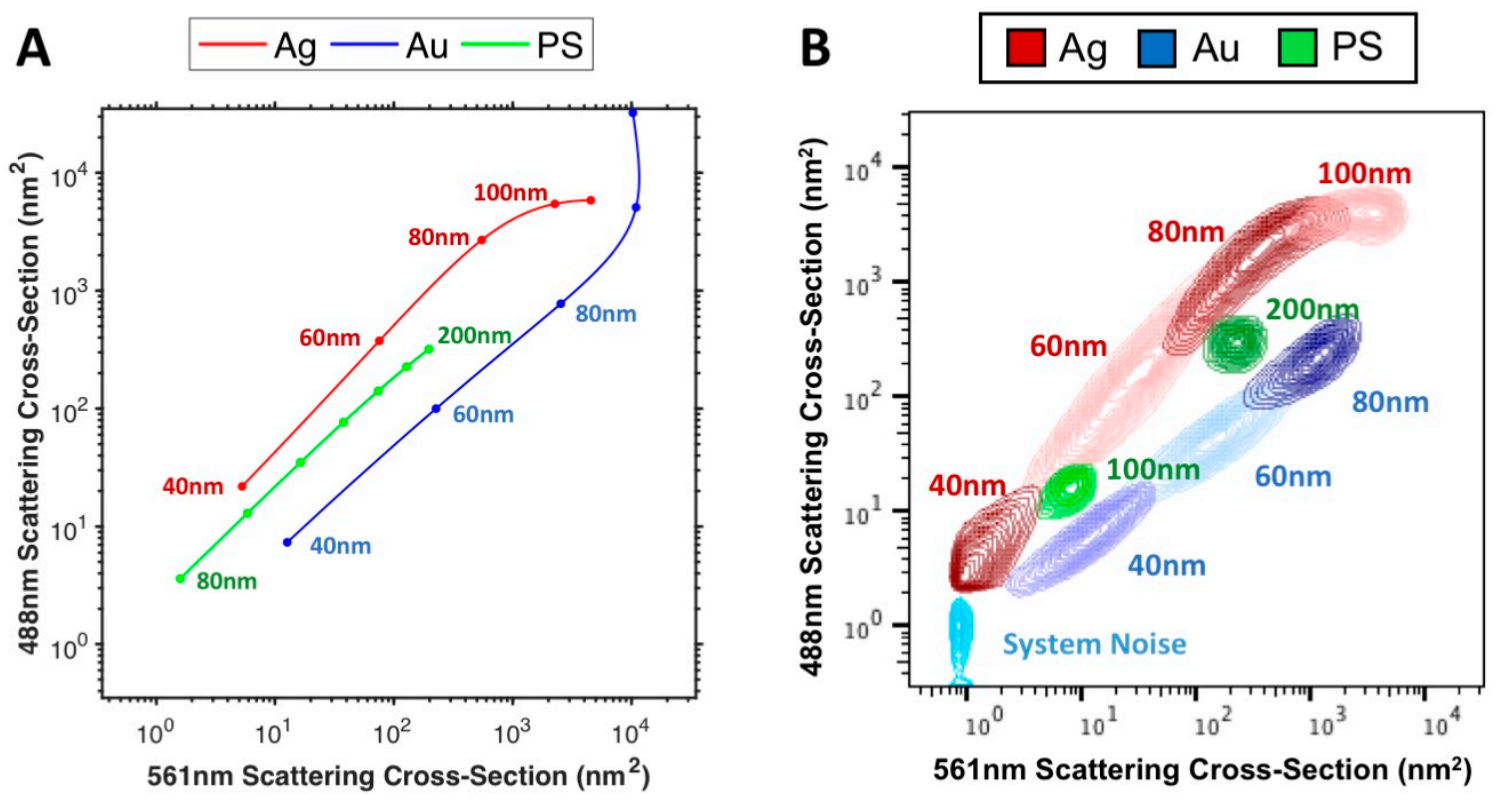

Figure 4. Multi-wavelength particle scattering models of the Astrios EQ (A) vs. acquired multi-wavelength particle scattering data on the Astrios EQ (B). Modelled data points are at diameters of $40 \mathrm{~nm}$ to $120 \mathrm{~nm}$ in $20 \mathrm{~nm}$ increments for $\mathrm{Au}$ and $\mathrm{Ag}$ and $80 \mathrm{~nm}$ to $200 \mathrm{~nm}$ in $20 \mathrm{~nm}$ increments for PS.

\subsection{A Simple Method of Deconvulsion of Labelled EVs versus Unabled EVs}

In order to implement the use of several labels at once, particles with unique spectral scattering properties would be required to provide a means of deconvolution. An example of two materials that show distinct spectral scattering properties are $60 \mathrm{~nm} \mathrm{Au}$ and Ag, Figure 5A. By plotting the ratio of $\mathrm{Ag}$ to $\mathrm{Au}$ scattering, the wavelengths at which the most separation is likely to occur can be anticipated. It can be seen that $60 \mathrm{~nm} \mathrm{Ag}$ particles will scatter more than $60 \mathrm{~nm}$ Au particles between the wavelengths of $350-510 \mathrm{~nm}$, before the inverse occurs from $510 \mathrm{~nm}$ to $800 \mathrm{~nm}$. This separation is confirmed in acquired data from the Astrios EQ which collects scatter at 405, 488, 561 and $640 \mathrm{~nm}$, Figure $5 \mathrm{~B}$, the relative power of this comparison not possible in this form however, where the detector voltages affect the acquired channel number. It can however be seen that the acquired $60 \mathrm{~nm} \mathrm{Ag}$ particles have a higher scattering intensity than $60 \mathrm{~nm} \mathrm{Au}$ at $405 \mathrm{~nm}$ and $488 \mathrm{~nm}$ before inverting on the $561 \mathrm{~nm}$ and $640 \mathrm{~nm}$ wavelengths, as predicted from modelling. 
The scattering properties of CFSE-stained EVs were then compared to those of $60 \mathrm{~nm} \mathrm{Au}$ and $60 \mathrm{~nm} \mathrm{Ag}$ particles, to show how their use as a label would enable distinction of epitope staining, Figure 5C. It can be seen that if CFSE-stained EVs were to be positively stained by an Ag NanoTag, their $488 \mathrm{~nm}$ and $561 \mathrm{~nm}$ SSC channel intensity would increase. If CFSE-stained EVs were to be positively stained by an Au NanoTag, mainly their $561 \mathrm{~nm}$ SSC channel intensity would increase. The characteristic channel intensities seen with $\mathrm{Au}$ and Ag NanoTags are distinct from one another and these differences provide a means for labeling two different epitopes or detecting two different EV-associated molecules in one assay. These results are expected based on the scattering properties of $\mathrm{Au}$ and $\mathrm{Ag}$ seen in Figures 2 and 4B.
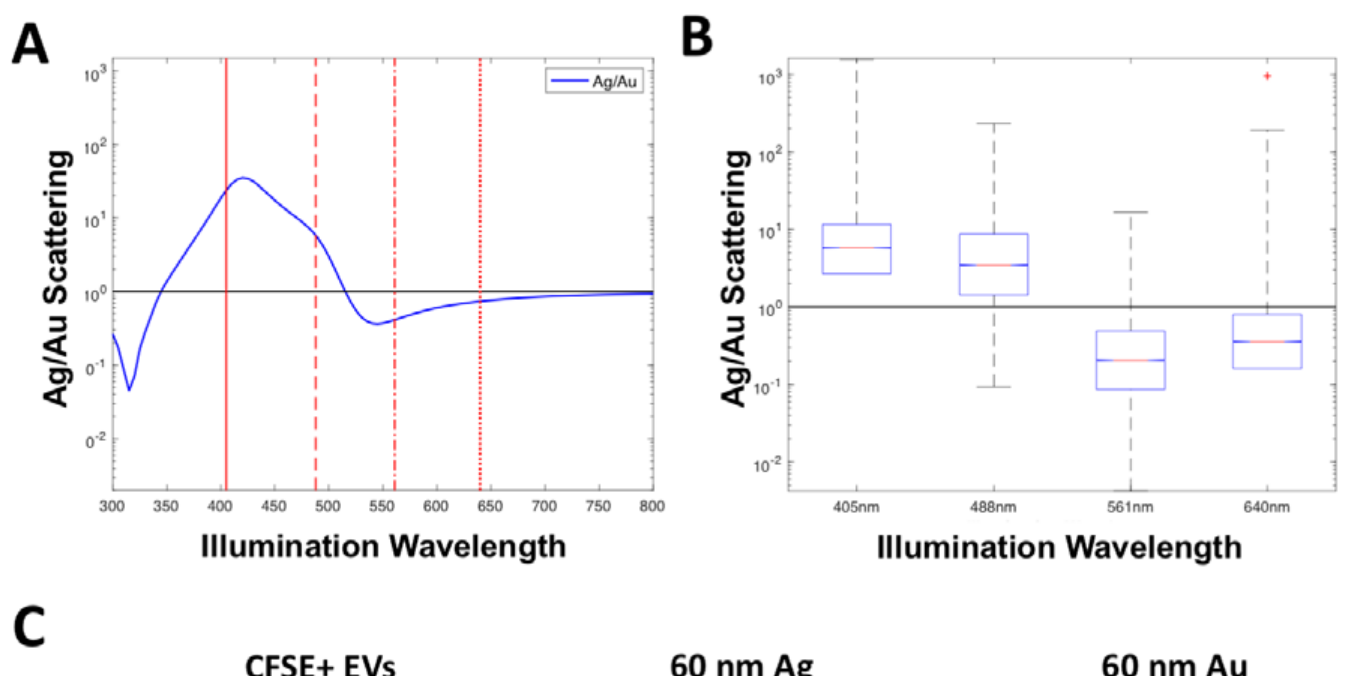

C

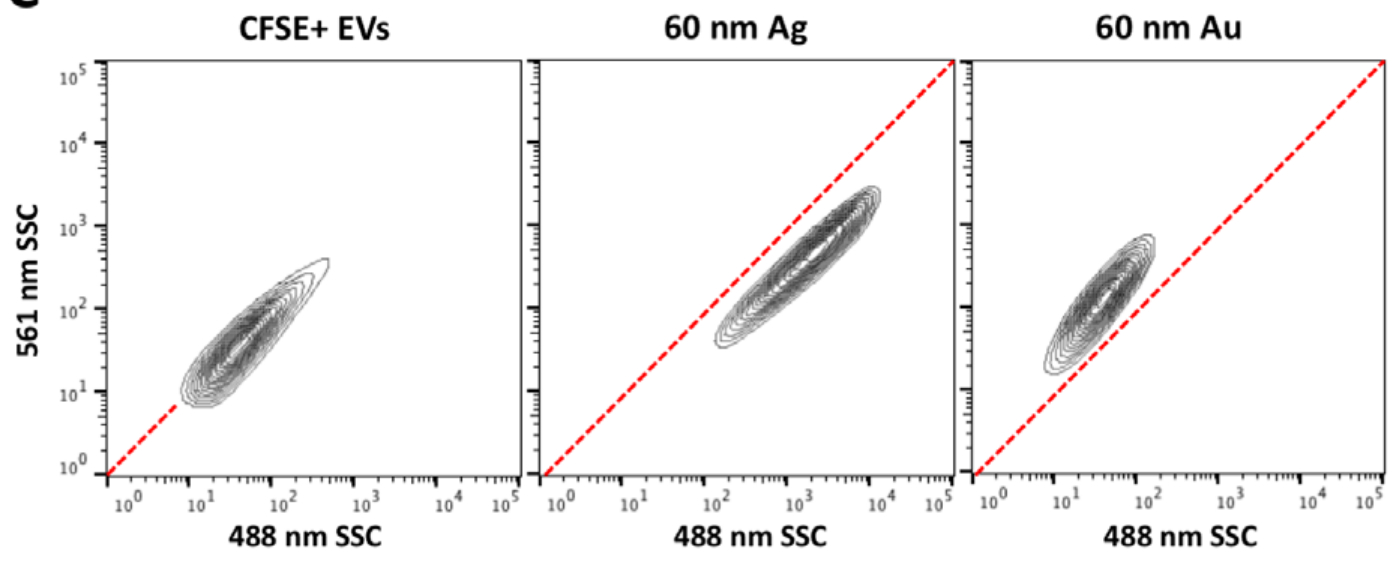

Figure 5. Simple spectral deconvulsion approaches to scattering labels. Illustrated is the predicted scattering cross-section ratio of $60 \mathrm{~nm}$ Ag to $60 \mathrm{~nm}$ Au particles (A) and acquired ratio of Ag to Au (B). Vertical red lines represent the particle scattering wavelengths $(405,488,561,640 \mathrm{~nm})$ collected on the Astrios EQ instrument. The acquired scattering channel data for CFSE-stained EVs, $60 \mathrm{~nm} \mathrm{Ag}$ and $60 \mathrm{~nm}$ Au particles on $488 \mathrm{~nm}$ and $561 \mathrm{~nm}$ SSC channels (C). The green area indicates a higher scattering power at $561 \mathrm{~nm}$, while the red area indicates a higher scattering power at $488 \mathrm{~nm}$. The red dotted line indicates an equal scattering power at $561 \mathrm{~nm}$ and $488 \mathrm{~nm}$.

\section{Discussion}

We have shown that particles with diameters of 40,60 and $80 \mathrm{~nm}$, composed of Ag or Au, can be partially or fully resolved by Astrios EQ and FACS Symphony flow cytometers. However, these cytometers may be considered 'high-end' pieces of equipment and not all conventional flow cytometers are capable of detecting the $100 \mathrm{~nm}$ polystyrene spheres used as a reference standard in our work here. While we have demonstrated that our flow cytometers are capable of detecting $40 \mathrm{~nm}$ particles at 
multiple scattering detection wavelengths, an ideal instrument for analysis of multiple ( $>3)$ spectral scatter labels simultaneously would utilize a broad range of illumination wavelengths; this could be in the form of a supercontinuum white laser, as well as multiple scatter detection channels. While using just one or two labels is feasible using a conventional flow cytometer configuration with scattering collection filters.

Due to the limited surface area and therefore surface proteins on EVs, steric hindrance is likely a problem for current immunoglobulin labels, which are approximately $15 \mathrm{~nm}$ in diameter. While this is an issue, especially for an immunoglobulin labelled with a few fluorophores that are undetectable on current instruments, only one NanoTag need bind to an EV surface epitope for it to be detectable. A $100 \mathrm{~nm}$ EV has the capacity to bind 20 loosely packed $15 \mathrm{~nm}$ spherical particles. Concentrations of NanoTags to EVs could therefore be far lower, not saturating EV surfaces, thereby allowing multiple NanoTags targeted to different proteins to bind. Another important factor when considering the use of nanoparticles as labels is their conjugation. Many conventional nanoparticle labels, such as Qdots, result in polyvalent labels. To ensure single NanoTag binding to EVs the development of monovalent labels would be instrumental. Monovalent labelling of Qdots has previously been demonstrating by wrapping DNA around the nanoparticle [6]. This method leaves a single functionalized end group that can be used to bind antibodies, aptamers, and so forth. A priority of future work to develop NanoTags as a viable labelling method would need to focus on creating a monovalent labelling methodology such as this.

As single NanoTags are capable of being detected without being bound to a targeted particle, such as an EV, a method of distinguishing labeled from unlabeled EVs are is required. One approach is to fluorescently label all EVs with an intercalating membrane dye, or non-specific stain such as CFSE [16]. It would then be possible to identify a shift in a NanoTag's fluorescence when bound to a labelled $\mathrm{EV}$, to determine whether it has labelled an EV or not. Alternatively, if a specific type of EVs were of interest for example, phosphatidylserine positive, it would be possible to make a NanoTag specific for the population of interest and use a second NanoTag targeted at an EV subset for example, CD9 positive. A unique scattering distribution would then occur for EVs that had two NanoTags, each with distinct scattering properties, bound to them.

In summary, single 40,60 and $80 \mathrm{~nm} \mathrm{Au}$ and $\mathrm{Ag}$ particles are not only detectable using conventional forms of flow cytometry but are also uniquely distinguishable from one another and fluorescently-labelled EVs, by using their spectral scattering properties. It is feasible that particles of such diameter and composition could be used as polyvalent or even monovalent detection labels, using currently available labelling methods, as means for single-epitope detection and implemented as single-epitope detection tags.

Our proposal for Molecular NanoTag as labels leverages the light scattering properties of high refractive index or highly plasmonic nano-materials, thereby providing a signal high enough for single molecules detection using currently available flow methodologies and may therefore be useful as labels for low expression, low scatter targets, such as EVs.

There are two main applications where Molecular NanoTags fill an existing gap in available detection methods. First, there are no methods currently available whereby a clinical laboratory is able to take a blood sample at and determine how many EVs are positive for a specific tumor marker per unit volume of blood. Rather current methods are only able to bind EVs to multi-micron sized beads and use detection antibodies to detect the EVs bound to the larger beads. Since individual Molecular NanoTags are labels that can be resolved individually, the detection of EVs with as few as one Molecular Nanotag-labelled receptor is feasible. Second, assembly of the Molecular NanoTags in a manner that ensures that the labels are monovalent enables enumeration of the number of labeled molecules. Such molecular enumeration is a significant advancement beyond the enumeration capabilities of current flow cytometric labels and instruments. 


\section{Patents}

Jones JC, et al., “Molecular NanoTags”. Provisional U.S. Patent Application No. 62/411,324; PCT Patent Application No. PCT/US2017/057928 filed 23 October 2017.

Supplementary Materials: The following are available online at http:/ /www.mdpi.com/1424-8220/18/8/2461/s1, Figure S1: Refractive index (A) and extinction coefficient (B) of zirconium, titanium dioxide, platinum, lead, iron oxide, copper, cadmium selenide, gold, and silver at wavelengths between 300-800 nm.; Figure S2: Serial dilutions of $40 \mathrm{~nm}(\mathrm{~A}, \mathrm{~B}), 60 \mathrm{~nm}(\mathrm{C}, \mathrm{D})$, and $80 \mathrm{~nm}(\mathrm{E}, \mathrm{F})$ Au nanoparticles.; Figure S3: Modelling data of each detection wavelength.; Figure S4: Modelled scattering cross-sections assuming Astrios EQ collection optics.; Figure S5: Comparison of the scattered optical power at $488 \mathrm{~nm}$ wavelength for gold, silver, and polystyrene nanoparticles measured on Astrios EQ and FACS Symphony flow cytometers in the forward (FSC) and sideward (SSC) direction. Figure S6: Multi-wavelength particle scattering models of the Astrios EQ vs. acquired multi-wavelength particle scattering data on the Astrios EQ.

Author Contributions: J.C.J., A.R. and J.A.W. contributed to the conceptual design of the Molecular NanoTag approach and designed the experiments and mathematical models presented herein. J.A.W. and J.C.J. wrote the manuscript. J.A.W. performed the mathematical models and experiments shown. J.K. and A.R. assisted with the experiments and analysis. J.A.W., J.K., A.R., P.H., J.A.B. and J.C.J. contributed to the data analysis, interpretation and manuscript revisions.

Funding: This work was supported by the U.S. National Institutes of Health, National Cancer Institute, Assistant Clinical Investigator Program.

Acknowledgments: Gold and silver nanosphere samples were provided by Cytodiagnostics. All experiments were performed in the NCI Vaccine Branch Flow Cytomtry Core Lab, with technical and logistical support from the Core Lab manager, Katherine McKinnon.

Conflicts of Interest: The authors declare no conflict of interest.

\section{References}

1. van der Pol, E.; Boing, A.N.; Gool, E.L.; Nieuwland, R. Recent developments in the nomenclature, presence, isolation, detection and clinical impact of extracellular vesicles. J. Thromb. Haemost. 2016, 14, 48-56. [CrossRef] [PubMed]

2. Bendall, S.C.; Nolan, G.P.; Roederer, M.; Chattopadhyay, P.K. A deep profiler's guide to cytometry. Trends. Immunol. 2012, 33, 323-332. [CrossRef] [PubMed]

3. Nolan, J.P. Flow Cytometry of Extracellular Vesicles: Potential, Pitfalls, and Prospects. Curr. Protoc. Cytom. 2015, 73, 1-16.

4. Chattopadhyay, P.K.; Price, D.A.; Harper, T.F.; Betts, M.R.; Yu, J.; Gostick, E.; Perfetto, S.P.; Goepfert, P.; Koup, R.A.; De Rosa, S.C.; et al. Quantum dot semiconductor nanocrystals for immunophenotyping by polychromatic flow cytometry. Nat. Med. 2006, 12, 972-977. [CrossRef] [PubMed]

5. Welsh, J.A.; Holloway, J.A.; Wilkinson, J.S.; Englyst, N.A. Extracellular Vesicle Flow Cytometry Analysis and Standardization. Front. Cell Dev. Biol. 2017, 5, 78. [CrossRef] [PubMed]

6. Farlow, J.; Seo, D.; Broaders, K.E.; Taylor, M.J.; Gartner, Z.J.; Jun, Y.W. Formation of targeted monovalent quantum dots by steric exclusion. Nat. Methods 2013, 10, 1203-1205. [CrossRef] [PubMed]

7. Francis, J.E.; Mason, D.; Levy, R. Evaluation of quantum dot conjugated antibodies for immunofluorescent labelling of cellular targets. Beilstein J. Nanotechnol. 2017, 8, 1238-1249. [CrossRef] [PubMed]

8. Banerjee, A.; Grazon, C.; Pons, T.; Bhatia, D.; Valades-Cruz, C.A.; Johannes, L.; Krishnan, Y.; Dubertret, B. A novel type of quantum dot-transferrin conjugate using DNA hybridization mimics intracellular recycling of endogenous transferrin. Nanoscale 2017, 9, 15453-15460. [CrossRef] [PubMed]

9. McPeak, K.M.; Jayanti, S.V.; Kress, S.J.P.; Meyer, S.; Iotti, S.; Rossinelli, A.; Norris, D.J. Plasmonic Films Can Easily Be Better: Rules and Recipes. ACS Photonics 2015, 2, 326-333. [CrossRef] [PubMed]

10. Querry, M.R. Optical Constants; Academic Press: Cambridge, MA, USA, 1985.

11. Siefke, T.; Kroker, S.; Pfeiffer, K.; Puffky, O.; Dietrich, K.; Franta, D.; Ohlidal, I.; Szeghalmi, A.; Kley, E.B.; Tunnermann, A. Materials Pushing the Application Limits of Wire Grid Polarizers further into the Deep Ultraviolet Spectral Range. Adv. Opt. Mater. 2016, 4, 1780-1786. [CrossRef]

12. Werner, W.S.M.; Glantschnig, K.; Ambrosch-Draxl, C. Optical Constants and Inelastic Electron-Scattering Data for 17 Elemental Metals. J. Phys. Chem. Ref. Data 2009, 38, 1013-1092. [CrossRef] 
13. Windt, D.L.; Cash, W.C.; Scott, M.; Arendt, P.; Newman, B.; Fisher, R.F.; Swartzlander, A.B. Optical-Constants for Thin-Films of Ti, Zr, Nb, Mo, Ru, Rh, Pd, Ag, Hf, Ta, W, Re, Ir, Os, Pt, and Au from 24-a to 1216-A. Appl. Optics 1988, 27, 246-278. [CrossRef] [PubMed]

14. Sultanova, N.; Kasarova, S.; Nikolov, I. Dispersion Properties of Optical Polymers. Acta Phys. Pol. A 2009, 116, 585-587. [CrossRef]

15. Daimon, M.; Masumura, A. Measurement of the refractive index of distilled water from the near-infrared region to the ultraviolet region. Appl. Opt. 2007, 46, 3811-3820. [CrossRef] [PubMed]

16. Morales-Kastresana, A.; Telford, B.; Musich, T.A.; McKinnon, K.; Clayborne, C.; Braig, Z.; Rosner, A.; Demberg, T.; Watson, D.C.; Karpova, T.S.; et al. Labeling Extracellular Vesicles for Nanoscale Flow Cytometry. Sci. Rep. 2017, 7, 1878. [CrossRef] [PubMed]

17. van der Pol, E.; van Gemert, M.J.; Sturk, A.; Nieuwland, R.; van Leeuwen, T.G. Single vs. swarm detection of microparticles and exosomes by flow cytometry. J. Thromb. Haemost. 2012, 10, 919-930. [CrossRef] [PubMed]

18. Matzler, C. MATLAB Functions for Mie Scattering and Absorption; University of Bern: Bern, Switzerland, 2002.

19. Fattaccioli, J.; Baudry, J.; Émerard, J.-D.; Bertrand, E.; Goubault, C.; Henry, N.; Bibette, J. Size and fluorescence measurements of individual droplets by flow cytometry. Soft Matter 2009, 5, 2232. [CrossRef]

(C) 2018 by the authors. Licensee MDPI, Basel, Switzerland. This article is an open access article distributed under the terms and conditions of the Creative Commons Attribution (CC BY) license (http:/ / creativecommons.org/licenses/by/4.0/). 